\title{
NONTHERMAL AND THERMAL EMISSIONS IN SOLAR FLARES
}

\author{
Nasiaki Nitta \\ Astronomy Program, University of Maryland, College Park, MD 20742, U.S.A.
}

\begin{abstract}
In two major solar flares, we have found evidence for hard X-ray footpoint emission using the $\mathrm{X}$-ray imager on Hinotori. Possible factors are discussed as to why we only rarely detect footpoint cmissions, which may not always take the form of double sources, depending on magnetic configurations in tlares.
\end{abstract}

\section{Introduction}

It is often argued that, in a majority of solar flares (so-called "type B" flares), hard X-rays emanate from conjugate footpoints of a sheared loop or loop system, emitted by a symmetric electron beam which precipitates into the chromosphere and interacts with the ambient plasma (see Tanaka 1983; Tsuneta 1984). However, apart from earlier analyses (Hoyng et al. 1981; Duijveman et al. 1982) with the Hard X-ray Imaging Spectrometer (HXIS: the resolution 8") on the Solar Maximum Mission (SMM), double hard X-ray sources are far from common (Duijveman and Hoyng 1983). This is more so in observations with the Solar X-ray Telescope (SXT: the resolution $\gtrsim 15$ ") on Hinotori, where hard $\mathrm{X}$-ray images usually show a single source (Takakura et al. 1983, 1984).

However, through further analyses of Hinotori flares, we now come to believe that, under favorable conditions, footpoint emission can be detected even with SXT. In this report, we present two of such examples and discuss what are distinct in these flares.

\section{Flare on 1982 February 3}

This flare is described in detail elsewhere (Nitta et al. 1989). Hard X-ray time profiles consist of two major parts (01:05 01:13 UT and 01:14 01:23 UT) separated by a deep valley. In Fig. 1, we show both hard X-ray (dotted lines, $27 \sim 54 \mathrm{keV}$ ) ind soft X-ray (solid lines, approximately $5 \sim 10 \mathrm{keV}$ ) images on nearly simultaneous $\mathrm{H} \alpha$ filtergrams. At 01:10:15 UT, corresponding to a peak in the first part, hard X-ray emission comes from two locations whereas soft X-ray emission emanates from in between them. We associate the two hard X-ray emitting regions with footpoints of a loop system (which is similar to $\mathrm{H} \alpha$ post flare loops seen in Fig. 1(d) well after hard X-rays decay) and the soft X-ray source with the loop top. The small displacement of the hard X-ray sources with respect to the brightest $\mathrm{H} \alpha$ patches can be attributed to projection effect and/or possible errors ( $\left.\$ 10^{\prime \prime}\right)$ in coalignment of the images.

In Fig. 1(b), obtained at a peak of the second part (01:14:10 UT), only one footpoint is seen. About three minutes after the last hard $\mathrm{X}$-ray peak, hard $\mathrm{X}$-ray emission assumes a single source (Fig. 1(c)), nearly cospatial with soft $\mathrm{X}$-ray emission which comes from the loop top all the time during the period covered by in Figs. 1(a) (c). We believe that the hard X-ray source in this phase is due to thermal emission of temperature $\gtrsim 3 \times 10^{7} \mathrm{~K}$, similar to the one observed by Lin et al. (1981), since the spectrum at low energies is very soft. 
(a)

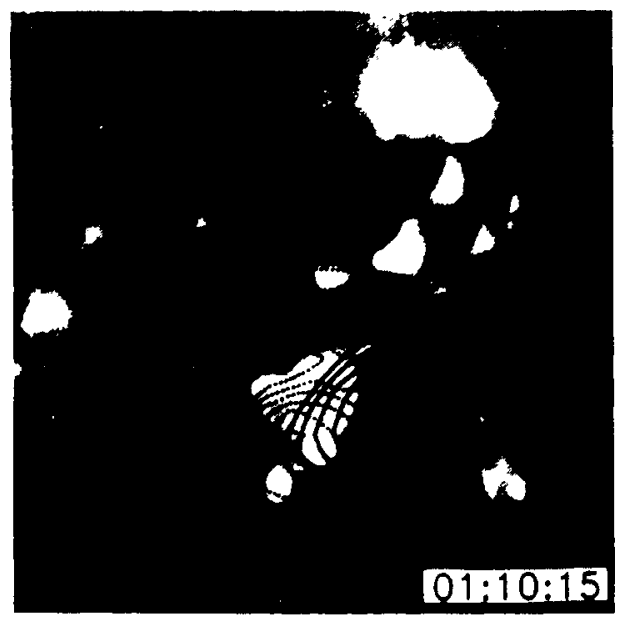

(b)

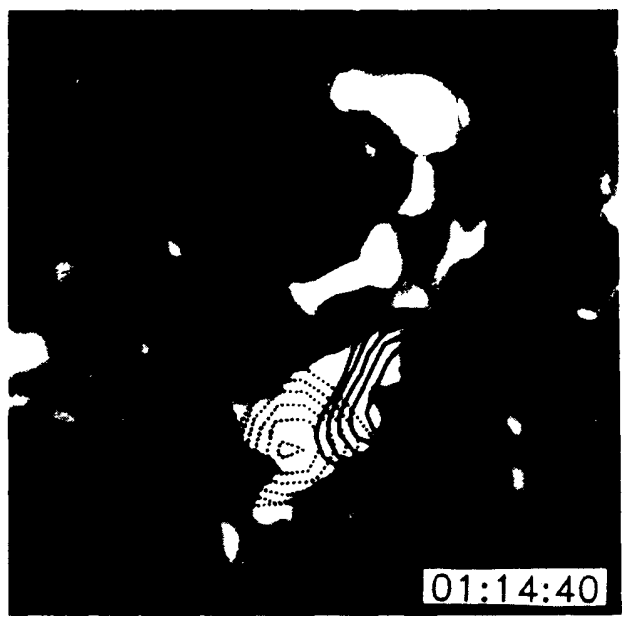

1

(d)

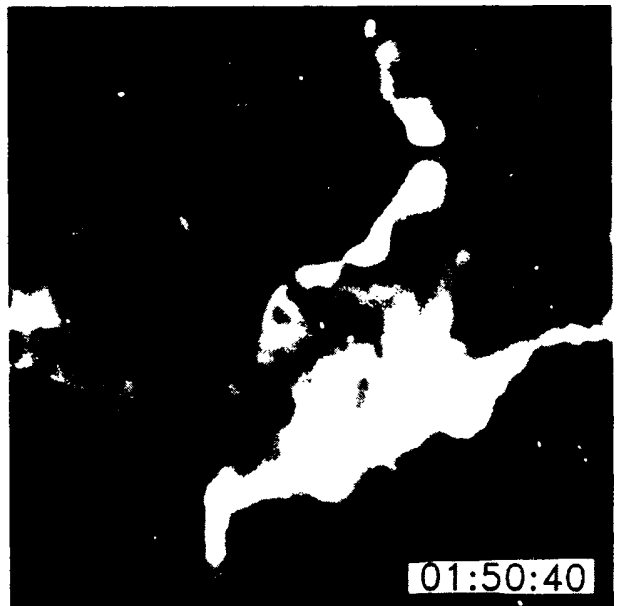

Fig. 1- Overlay of hard X-ray (dotted lines) and soft X-ray (solid lines) inages on $\mathrm{Ha}$ filtergrams at peaks ((a) and (b)) and in the decay phase ((c)). Contour levels are $15.9 \%, 22.5 \%, 31.8 \%, 45 \%, 63.6 \%$ and $90 \%$ of the peak intensity in each image. North is at the top and west is to the right. An $\mathrm{H} \alpha$ filtergram in the post flare period is shown in (d).

\section{Flare on 1981 October 15}

There is again evidence for footpoint emission for this flare. Hard X-ray (approximately $25 \sim 50 \mathrm{keV}$ ) images (Figs. 2(a) (c)) change significantly in about 1 min. At first only one footpoint $\left(A_{1}\right)$ is seen. Just after the first peak at 04:44:07 UT, a second source (B) appears, as $A_{1}$ moves southward (or rather, a new footpoint $A_{2}$ emerges). Then another footpoint $A_{3}$ dominates. In Fig. 2(d), the Kitt Peak magnetogram (with potential field lines) at 16:33 UT on the same day is shown. Magnetic features are shifted unforshortened to allow for the rotation of the Sun. The SXT field of view is drawn for reference. Comparison with hard $\mathrm{X}$-ray images suggests that footpoints $A_{1}, A_{2}$ and $A_{3}$ are all of positive polarity while the footpoint $B$ is of negative polarity. 
This flare is unusual in the sense that soft $\mathrm{X}$-ray emission is very weak with respect to hard $X$-ray emission (Fig. 3). Soft $X$-ray images are not available. Since the spectrum in $10 \sim 30 \mathrm{keV}$ ranges is not affected much by thermal emission of temperatures $(1.5 \sim 2.5) \times 10^{7} \mathrm{~K}$ which is commonly observed in solar flares, this flare offers a rare chance to study the extension of nonthermal spectrum to low energies (Nitta and Dennis 1988).

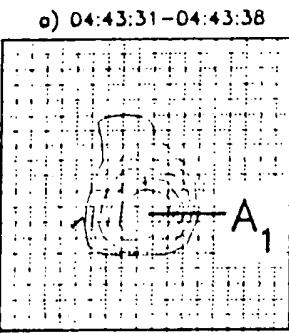

D) $04: 44: 16-04: 44: 23$

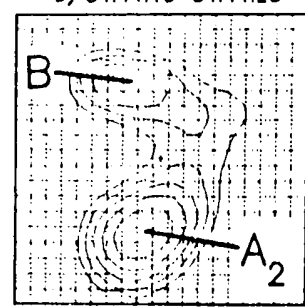

c) $04: 44: 42-04: 44: 49$

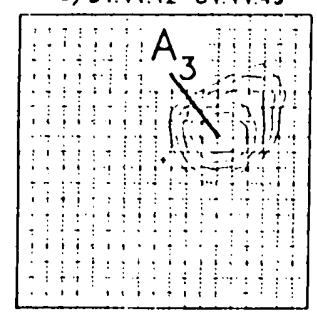

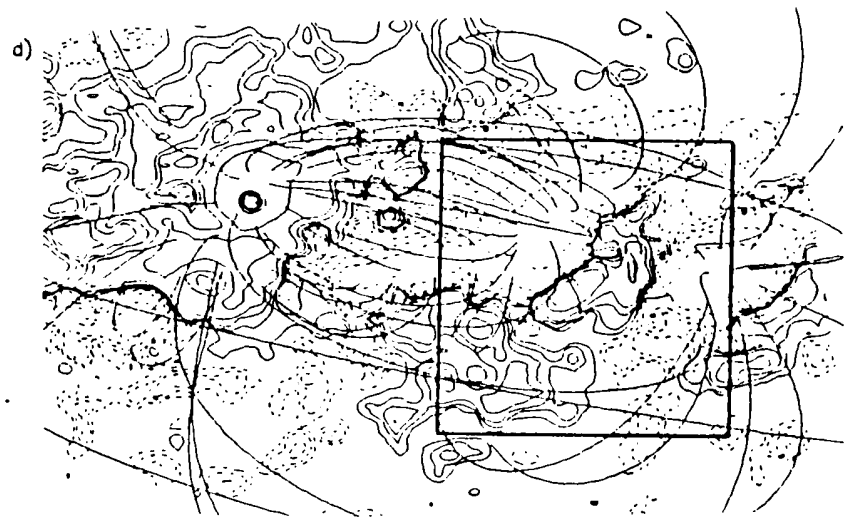

Fig 2- (a) (c) Hard X-ray images for the flare on 1981 October 15 at three representative times. Contour levels are $15.9 \%, 22.5 \%, 31.8 \%, 45 \%$ and $63.6 \%$ of the peak intensity in each image. (d) Kitt Peak magnetogram at 16:33 UT on the same day (solid lines for positive polarity and dashed lines for negative polarity), with potential field lines. The SXT field of view is drawn for reference.

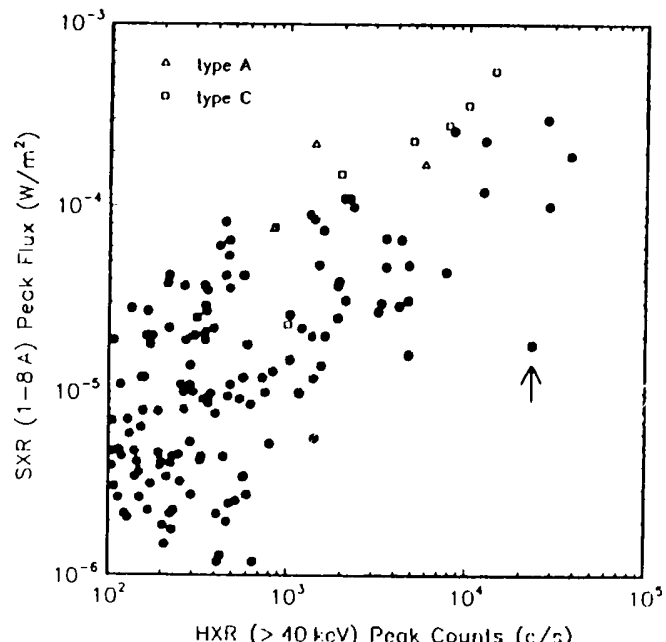

Fig. 3- Peak soft $\mathrm{X}$-ray flux vs peak hard $\mathrm{X}$-ray flux for 147 Hinotori flares. The 1981 October 15 flare is indicated by an arrow. Those flares showing clear spectral hardening over the peaks are classified as "type C" (7), while those with an extremely steep spectrum and small source size are classified as "type $A$ " (3). 


\section{Discussion}

Unlike previous analyses, we have been able to see footpoint emission with the Hinotori imager, which has a spatial resolution of only $\gtrsim 15$ ". Wide separation of footpoints (50" 70"), commonly observed in both of the two flares, is certainly a key factor for detection of footpoint emission, although it is not well known how separated footpoints of flare loops generally are. Without comparisons of $\mathrm{X}$-ray inages with optical informaion such as $\mathrm{Ha}$ pictures and manetograms, which have not been made systematically in other flares, the fact that hard $\mathrm{X}$-ray images show a single source cannot rule out the possibility of footpoint emission, since footpoint emission often takes the form of a single source as Figs. 1(b), 2(a) and 2(c) indicate. We consider that flare loops are more or less asymmetric (see Maetzler 1976; Kundu and Vlahos 1979), letting electrons precipitate nearly freely at the footpoint of weaker magnetic field, while mirroring them back above the footpoint of stronger magnetic field. Both exaunles shown here have stronger (weaker) hard X-ray emission on plage (sunspot) regions.

For the 1982 February 3 flare, although coalignment of X-ray images on $\mathrm{H} \alpha$ filtergrams leaves some uncertainty, simultaneous soft $X$-ray images, which are doninated by thermal emission of temperatures $\sim 2 \times 10^{7} \mathrm{~K}$ have been useful in associating hard X-ray emission with footpoints. For the 1981 October 15 flare, exceptionally small soft X-ray flux (Fig. 3) may lead us to observe footpoint cuissions. In fact the low energy ( $\$ 40 \mathrm{keV})$ hard X-ray spectrum shows a clear flattening toward low energies (Nitta and Dennis 1988). This in turn suggests that what is observed to be a power-law spectrum may be decomposed into a flattening nonthermal spectrum superposed by thermal emission (whose temperature should be considerably higher than $2 \times 10^{7} \mathrm{~K}$ ). If this is the case, some, if not all, of the hard X-ray images so far obtained at $\lesssim 35 \mathrm{keV}$ ) may be affected significantly by the thermal components.

The author acknowledges support from NSF grant ATM 87-17157, NASA contract NAG 5-969 and NASA grant NGR 21-002-199 to the University of Maryland.

\section{References}

Duijvernan, A., Hoyng, P., and Machado, M.E. 1982, Solar Phys., 81, 137.

Duijveman, A. and Hoyng, P. 1983, Solar Phys., 86, 279.

Hoyng, P., Duijveman, A., Machado, M.E., Rust, D.M., and Svestka, Z. 1981, Ap.J.(Lettery), 268, L155.

Kundu, M.R. and Vlahos, L. 1979, Ap.J., 232, 595.

Lin, R.P., Scliwartz, R.A., Pelling, R.M., and Hurley, K.C. 1981, Ap.J.(Letters), 251,L109.

Mactzler, C. 1976, Solur Phys., 49, 117.

Nitta, N., Kiplinger, A.L., and Kai, K. 1989, Ap.J., in press.

Nitta, N. and Dennis, B.R. 1988, in preparation.

Takakura, T., Ohki, K., Tsuneta, S., and Nitta, N. 1983, Solar Phys., 86, 323.

Takakura, T., Tanaka, K., and Hiei, E. 1984, Adv. Space Res., 4, 143.

Tanaka, K. 1983, in IAU Colloq. 71, Activity in Red Dwarf Stars, eds. P.B. Byrne and M. Rodono, (Dordrecht: Reidel), p.307.

Tsuneta, S. 1984, in Proc. Japan-France Joint Seminar on Active Phenomena in the Outer Atmosphere of the Sun and Stars, ed. J.C. Pecker and Y. Uchida (Paris: CNRS and L'Observation de Paris). 